\title{
Evaluación de una unidad de cirugía de día
}

\author{
Gilda Verónica Chirigliano ${ }^{1}$ y María Cristina Noceti ${ }^{1}$
}

RESUMEN Objetivos. Presentar una evaluación de la Unidad de Cirugía del Día (UCD) del Centro de Asistencia del Sindicato Médico del Uruguay (CASMU) de Montevideo, instalada en el Sanatorio 1 de esta institución, un hospital de 107 camas destinado antes a la actividad quirúrgica de mediana y baja complejidad. Se analizan los resultados del proceso de reorganización de la actividad quirúrgica bajo el régimen de la cirugía de día mediante indicadores estadísticos de utilización del servicio, costos y satisfacción del paciente.

Métodos. Se utilizaron las estadísticas asistenciales para determinar la cobertura del nuevo régimen, los días de internación, los costos anteriores y posteriores a la puesta en funcionamiento de la UCD y la opinión de los pacientes sobre la calidad de la atención.

Resultados. Tras los primeros 2 años de funcionamiento de la UCD (1998-1999), más de un tercio de las operaciones de las especialidades seleccionadas se realizaban en el régimen de cirugía de día. Dichas especialidades representan $84 \%$ del total de las actividades quirúrgicas realizadas por la UCD. Los días de estadía en las especialidades incluidas en el sistema disminuyeron en $26 \%$. El análisis de costo-efectividad de esta modalidad muestra resultados dos veces y media más favorables que la forma convencional. En general, los pacientes manifestaron gran satisfacción con los servicios prestados por la UCD.

Discusión. Los resultados obtenidos permiten recomendar definitivamente esta forma de atención, dado que es de buena calidad, logra la satisfacción del paciente y reduce radicalmente los costos, gracias a la disminución de la estadía y de los recursos necesarios en los servicios de internación. Existen posibilidades de expansión de este nuevo régimen mediante la incorporación de otros procesos patológicos y de las técnicas elegibles.

Palabras clave Cirugía ambulatoria, cirugía de día, costo-efectividad, gestión de servicios de salud.

El Centro de Asistencia del Sindicato Médico del Uruguay (CASMU), institución de asistencia médica colectiva de Montevideo, Uruguay, es un seguro de prepago con 265 mil afiliados (1996-1999) que cuenta con 462 camas de internación ubicadas en cuatro hospitales: los Sanatorios 1 y 2 , des-

\footnotetext{
1 Centro de Asistencia del Sindicato Médico del Uruguay (CASMU), Montevideo, Uruguay. La correspondencia debe ser enviada a Gilda Chirigliano a la siguiente dirección: Asilo 3336, Montevideo, Uruguay. Correo electrónico: tihista@adinet. com.uy
}

tinados fundamentalmente a la labor médico-quirúrgica, el Sanatorio 3 a la atención maternoinfantil y el Sanatorio 4 a los cuidados especiales e intensivos. En 1999, los egresos quirúrgicos fueron unos 37 por 1000 afiliados mayores de 15 años.

Desde comienzos de julio de 1997 el CASMU desarrolló una nueva modalidad de organización de los servicios quirúrgicos - la cirugía ambulatoria o cirugía de día- practicada habitualmente en otros países desde hacía años, como forma de lograr una atención quirúrgica de buena calidad y reducir los costos asistenciales gracias a un menor uso de las camas de internación.

La cirugía de día es aquella en la que al paciente se le puede dar de alta en 24 horas, sin utilizar camas de internación, ${ }^{2}$ e incluye procedimientos de cirugía menor y de mayor complejidad, realizados con anestesia local o general. Esta modalidad se empezó a utilizar tras un proceso de cambio de más

\footnotetext{
2 Gilardoni F. Informe sobre la realización de la Cirugía Ambulatoria en el CASMU. Comisión de Cirugía Ambulatoria. Centro de Asistencia del Sindicato Médico del Uruguay, Montevideo, 1990.
} 
de una década en el que participaron numerosos actores de diversos ámbitos. ${ }^{3}$ Inicialmente se limitó a determinadas especialidades, procesos y procedimientos que podrán ampliarse a corto y medio plazo. La descripción detallada del proceso de planificación de la Unidad de Cirugía de Día (UCD) del CASMU consta en un trabajo anterior. ${ }^{4}$

El Sanatorio 1 realizaba hasta el momento del cambio la mayor parte de las intervenciones quirúrgicas de baja y mediana complejidad, con lo cual concentraba un considerable volumen de actividad quirúrgica que era posible realizar de forma ambulatoria. El proceso de cambio hacia la cirugía de día también modificó la actividad quirúrgica global de la institución, incluida la realizada en los otros sanatorios. La disminución del número de camas de internación y de los costos sanatoriales era en ese momento un objetivo de la institución, constituyéndose en otro factor determinante de la puesta en marcha del proyecto.

Los procedimientos de las especialidades que operaban en el Sanatorio 1 y que eventualmente podían ser incluidos en un régimen de cirugía ambulatoria fueron seleccionados en 1996 por los departamentos quirúrgicos respectivos y comprendieron básicamente la cirugía general, la cirugía pediátrica, la urología, la otorrinolaringología (ORL) y la cirugía bucomaxilar. Posteriormente se agregaron intervenciones de especialidades que operaban en el Sanatorio 2 del CASMU: oftalmología, cirugía plástica y traumatología.

En el Sanatorio 1, el mayor volumen de intervenciones programadas correspondía hasta ese momento a la cirugía

\footnotetext{
3 Noceti MC, Masliah R, Irigaray A, Chirigliano G, Rosasco C, Fernández N. Comisión de Cirugía Ambulatoria. Memorandum a médicos cirujanos CASMU. Centro de Asistencia del Sindicato Médico del Uruguay, Montevideo, 1997.

Romay R, Chirigliano G, Harreche M, Trotchansky J, Wetstein J, Romano R, et al. Informe de Comisión sobre Anteproyecto de remodelación del Primer piso de Sanatorio 1 del CASMU. Centro de Asistencia del Sindicato Médico del Uruguay, Montevideo, 1995.

Harreche M. Plan de Cirugía Ambulatoria a nivel del Hospital Maciel del Ministerio de Salud Pública, Uruguay. Montevideo, 1995.

4 Chirigliano G, Noceti MC. Planificación de una unidad de Cirugía del día. Centro de Asistencia del Sindicato Médico del Uruguay. Montevideo, 2001.
}

general y a la urología, que representaban el 68\% de las intervenciones programadas, seguidas por la cirugía pediátrica y la ORL. Estas cuatro especialidades representaban el 95\% de las intervenciones programadas realizadas en ese sanatorio. No incluimos en este trabajo la cirugía de día obstétrica y ginecológica, por realizarse en una unidad sanatorial independiente, el Sanatorio 3.

Los objetivos fundamentales de este estudio consistieron en:

- Determinar el porcentaje de intervenciones realizadas mediante el nuevo régimen, que, de acuerdo con lo previsto, debería ser inicialmente de alrededor de un 30\% de las intervenciones en las especialidades seleccionadas.

- Evaluar el ahorro en días-cama de internación.

- Analizar retrospectivamente la relación costo-efectividad de la cirugía de día en comparación con la cirugía convencional.

- Obtener elementos para evaluar la calidad de la atención a los pacientes, midiendo su satisfacción con la asistencia prestada.

\section{MATERIALES Y MÉTODOS}

La UCD se instaló en el Sanatorio 1 del CASMU, el cual absorbió bajo esta nueva modalidad intervenciones que antes se realizaban sobre todo en este sanatorio, pero también algunas efectuadas en el Sanatorio 2 (cirugía plástica y oftalmológica). Si bien se trata de un nuevo programa asistencial asentado en el Sanatorio 1 del CASMU, implicó una reorganización de toda la actividad quirúrgica institucional (12 446 intervenciones en el ejercicio 19961997). Se procuró incorporar las especialidades e intervenciones que mejor se adaptaban en ese momento al nuevo régimen por razones técnicas, organizativas $\mathrm{u}$ otras.

El Sanatorio 1, con 107 camas, realizaba en dicho ejercicio el 33\% de las operaciones totales; el Sanatorio 2, con 143 camas, el 50\%, y el Sanatorio 3, materno-infantil, el 15\% del total.
Había un porcentaje muy pequeño de operaciones realizadas en sanatorios contratados externos a la institución. En los últimos 10 años anteriores al ejercicio 1997-98 la institución presentó un número estable de intervenciones quirúrgicas anuales de alrededor de 12 000, así como una dotación estable de camas y escasa variación de la población afiliada $(2,5 \%)$.

De las ocho especialidades que se integraron en este nuevo régimen, para este análisis se seleccionaron las cuatro de mayor grado de adaptación al nuevo modelo y, por ende, en las que se podría conseguir mayor número de intervenciones: cirugía general, cirugía plástica, oftalmología y ORL (cuadro 1).

Las estadísticas asistenciales de egresos e intervenciones se obtuvieron de los informes estadísticos anuales del Departamento de Registros Médicos ${ }^{5}$ anteriores y posteriores a la apertura de la UCD y se presentan con respecto a las especialidades incluidas en la nueva modalidad de trabajo y al total de las mismas. Con estos datos se calculó el porcentaje de intervenciones realizadas en el nuevo régimen en cada especialidad. La información sobre los días de internación, desglosada por especialidades, se obtuvo de la misma fuente. El ahorro de días de internación se calculó como la diferencia observada entre los períodos anterior y posterior a la creación de la UCD.

En el estudio de costos se utilizó el costo del día-cama de internación en las especialidades seleccionadas, calculado por el Departamento Contable del CASMU, y el costo de la atención de día en la UCD, calculado a partir de los datos presupuestarios de las correspondientes Unidades Ejecutoras del Sanatorio 1. ${ }^{6}$ En ambos casos se incluyeron los costos directos e indirectos. El sistema de información presu-

\footnotetext{
5 Departamento de Registros Médicos. Informes estadísticos anuales. Centro de Asistencia del Sindicato Médico del Uruguay, Montevideo, ejercicios 1995-96, 1996-97 y 1997-98.

6 Informe de Gestión Contable. Balance de la Unidad de Cirugía del Día del CASMU. Centro de Asistencia del Sindicato Médico del Uruguay, Montevideo, ejercicio 1998-99.
} 
CUADRO 1. Actividad de la Unidad de Cirugía de Día por especialidad. Centro de Asistencia del Sindicato Médico del Uruguay (CASMU), Montevideo, Uruguay, ejercicio 1998-1999

\begin{tabular}{lrr}
\hline & \multicolumn{2}{c}{ Intervenciones } \\
\cline { 2 - 3 } \multicolumn{1}{c}{ Especialidad } & \multicolumn{1}{c}{ No. } & $\%$ \\
\hline Cirugía general & 712 & 19,7 \\
Cirugía pediátrica & 168 & 4,7 \\
Cirugía plástica & 1202 & 33,3 \\
Oftalmología & 419 & 11,6 \\
Urología & 145 & 4,0 \\
Técnicas (endoscopia) & 52 & 1,4 \\
Otorrinolaringología & 209 & 5,8 \\
Traumatología & 36 & 1,0 \\
Cirugía bucomaxilar & 35 & 1,0 \\
Subtotal cirugía & 2978 & 82,5 \\
Tratamiento del dolor & 634 & 17,5 \\
$\quad$ Total & 3612 & 100,0 \\
\hline
\end{tabular}

puestaria del Sanatorio 1 comprende los gastos directos e indirectos de la “Unidad Ejecutora de Cirugía del Día", excepto los honorarios médicos, que se obtienen a través del sistema de información de liquidaciones de técnicos del Sanatorio 1. Se calculó el costo diario de la atención de los pacientes en la UCD y el costo de la totalidad de los pacientes atendidos en períodos anuales.

La relación costo-efectividad (1) se determinó calculando el costo real que supusieron los pacientes tratados en la UCD por día de atención ambulatoria en el período elegido y comparándolo con el que se hubiera generado en caso de que los mismos pacientes hubieran sido tratados el mismo año en el régimen de cirugía convencional. No se calcularon los costos transferidos al paciente por la recuperación en su domicilio, aunque se estudió la necesidad de seguimiento domiciliario por médico o enfermera.

La calidad de la atención percibida por el paciente se evaluó mediante una encuesta de satisfacción de usuarios de la unidad, ${ }^{7}$ que recogió opiniones

\footnotetext{
7 Operti R, Cavasin C. Encuesta de satisfacción de usuarios de la Unidad de Cirugía del Día del CASMU. Centro de Asistencia del Sindicato Médico del Uruguay, Montevideo, 1999.
}

sobre el ingreso, la atención médica, la enfermería, la comodidad, el funcionamiento global del servicio, etc. La encuesta fue realizada en 1999 por un equipo contratado ajeno a la institución. Se entrevistó telefónicamente una muestra representativa de 255 pacientes que habían sido operados en la UCD. Además se consideró la tasa de reingresos en el servicio de internación como indicador de posibles complicaciones y de la necesidad de seguimiento domiciliario.

\section{RESULTADOS}

Los egresos totales y los de la cirugía de día en el período 1996-97 al 1998-99 muestran que a medida que disminuyen los primeros aumentan los segundos. Así, mientras los primeros bajaron de 27561 en el ejercicio 96-97 a 24444 en el ejercicio 98-99 (reducción de $3117,11 \%$ ), los segundos aumentaron de 276 a 3645 en los mismos ejercicios, con un total 3612 procedimientos realizados.

Durante los dos ejercicios evaluados de forma completa (1997-98 y 199899), la cirugía de día representó el $24 \%$ del total de las intervenciones quirúrgicas realizadas en la institución (en torno a las 12000 operaciones anuales en los últimos 10 años anteriores a la creación de la UCD). En las especialidades evaluadas (cirugía general, cirugía plástica, oftalmología y ORL), que tuvieron 7872 egresos en 1996-97, el nuevo régimen alcanzó el $32 \%$ del total (2 542 intervenciones) en el ejercicio 98-99 (cuadro 1).

El número de operaciones programadas y realizadas en la UCD permite observar un cumplimiento del $80 \%$ de lo programado, con 2978 operaciones realizadas (cuadro 1) de un total de 3722 programadas, lo cual muestra la alta implantación de la modalidad.

Los días de estadía totales corresponden a los egresos de los sanatorios propios y contratados y fueron 145077 en el ejercicio de 1996-97, que se tomó como año base; en el ejercicio 1997-98 descendieron un 7,5\% (10 859 días menos) y en el ejercicio 1998-99 un 10,7\% (15 524 días menos); la estadía media pasó de 5,3 en el ejercicio anterior a la implantación de la UCD (96-97) a 5,7 en el ejercicio 98-99. Este ligero aumento de la estadía hospitalaria media ( 0,4 días) se explica por el hecho de que las intervenciones resueltas por la UCD (de complejidad menor y media) se excluyeran de las estadísticas hospitalarias totales a partir de la fecha de implantación de la UCD.

Los días de estadía de los egresos de las especialidades seleccionadas (cirugía general, cirugía plástica, oftalmología y ORL) y su disminución a partir de la instalación de la UCD constan en el cuadro 2. Los días de estadía de estas especialidades se presentan en el ejercicio 1996-97 y luego en los años siguientes. Los mismos fueron en las especialidades mencionadas de 29486 días de internación en 1996-97, un 12,5\% menos (3 690 días) en 1997-98 y un $14,2 \%$ menos (4 184 días) en 1998-99.

El valor del día-cama de internación en el Sanatorio 1 en 1998-99 fue de 412 dólares estadounidenses (\$US), y el de la cirugía de día, de \$US 392. Del análisis de las diferencias de costos entre las dos modalidades (costo total anual de la cirugía de día de \$US 1167376 en el ejercicio 1998-99, frente a una estimación de \$US 3067340 para la cirugía convencional en el mismo ejercicio) y aceptando que la calidad de las dos opciones es similar, se deduce que la nueva modalidad tiene una relación costo-efectividad dos veces y media mejor que la modalidad convencional (cuadro 3).

De acuerdo con la encuesta telefónica realizada a 255 pacientes tras el alta, la UCD no tiene problemas de acceso, obtiene una buena evaluación del confort de la sala, plena conformidad con los procedimientos técnicos y una alta valoración de la atención de los cirujanos. La relación con el cirujano fue muy buena o excelente para $67 \%$ de los encuestados, $88,9 \%$ dijo tener una noción exacta de la intervención quirúrgica que se le practicó y 96,9\% manifiestó plena satisfacción con el tratamiento realizado. Además, los encuestados estarían dispuestos a hacer nuevamente uso del servicio. 
CUADRO 2. Egresos y días de estadía en las especialidades quirúrgicas seleccionadas. Centro de Asistencia del Sindicato Médico del Uruguay (CASMU), Montevideo, Uruguay, ejercicios 1996-97 a 1998-1999

\begin{tabular}{|c|c|c|c|c|c|c|}
\hline \multirow[b]{2}{*}{ Ejercicio } & \multicolumn{2}{|r|}{ Egresos } & \multicolumn{2}{|c|}{ Días de estadía } & \multicolumn{2}{|c|}{ Estadía media (días) } \\
\hline & No. & $\begin{array}{c}\text { Diferencia } \\
\text { con año base }\end{array}$ & No. & $\begin{array}{c}\text { Diferencia } \\
\text { con año base }\end{array}$ & Días & $\begin{array}{c}\text { Diferencia } \\
\text { con año base }\end{array}$ \\
\hline \multicolumn{7}{|c|}{ Cirugía general } \\
\hline $96-97$ & 4953 & & 24270 & & 4,9 & \\
\hline $97-98$ & 3872 & -1081 & 22844 & -1426 & 5,9 & 1,0 \\
\hline $98-99$ & 4198 & -755 & 23089 & -1181 & 5,5 & 0,6 \\
\hline \multicolumn{7}{|c|}{ Cirugía plástica } \\
\hline $96-97$ & 1542 & & 2621 & & 1,7 & \\
\hline $97-98$ & 564 & -978 & 1297 & -1324 & 2,3 & 0,6 \\
\hline $98-99$ & 301 & -1241 & 873 & -1748 & 2,9 & 1,2 \\
\hline \multicolumn{7}{|l|}{ Oftalmología } \\
\hline $96-97$ & 593 & & 949 & & 1,6 & \\
\hline $97-98$ & 283 & -310 & 566 & -383 & 2,0 & 0,4 \\
\hline $98-99$ & 121 & -472 & 327 & -622 & 2,7 & 1,1 \\
\hline \multicolumn{7}{|c|}{ Otorrinolaringología } \\
\hline $96-97$ & 784 & & 1646 & & 2,1 & \\
\hline $97-98$ & 519 & -265 & 1089 & -557 & 2,1 & 0,0 \\
\hline $98-99$ & 533 & -251 & 1013 & -633 & 1,9 & $-0,2$ \\
\hline \multicolumn{7}{|l|}{ Total } \\
\hline $96-97$ & 7872 & & 29486 & & 3,7 & \\
\hline $97-98$ & 5238 & -2634 & 25796 & -3690 & 4,9 & 1,2 \\
\hline 98-99 & 5153 & -2719 & 25302 & -4184 & 4,9 & 1,2 \\
\hline
\end{tabular}

CUADRO 3. Indicadores de utilización y costos de dos modalidades de atención quirúrgica, la cirugía de día y la cirugía convencional (estimado), en las especialidades seleccionadas. Centro de Asistencia del Sindicato Médico del Uruguay (CASMU), Montevideo, Uruguay, ejercicio 1998-1999

\begin{tabular}{lcc}
\hline Indicador de utilización de servicios y costos & Cirugía de día & Cirugía convencionala $^{\text {a }}$ \\
\hline Promedio estadía & 1 & 2,5 \\
No. de intervenciones quirúrgicas & 2978 & 2978 \\
No. de días de atención & 2978 & 7445 \\
Costo por día de atención (\$US) & 392 & 412 \\
Costo total atención (\$US) & 1167376 & 3067340
\end{tabular}

a Estimaciones realizadas suponiendo que los mismos enfermos sometidos a cirugía de día hubieran sido operados el mismo año mediante cirugía convencional.

\$US: dólares estadounidenses.

Las necesidades de seguimiento domiciliario fueron muy bajas ( $2 \%$ por médicos y $1 \%$ por enfermeras), al igual que el porcentaje de reingresos $(1 \%)$.

\section{DISCUSIÓN}

La cirugía de día se ubica en el área de las nuevas tecnologías de salud $\mathrm{y}$ de las innovaciones en las formas aplicación de modelos normativos teóricos que toman como base la opinión del usuario de los servicios (2). En estos trabajos se entiende como satisfacción del paciente el grado en que la atención brindada alcanza sus expectativas y preferencias, criterio que se usó en la evaluación de esta unidad de atención.

Existen experiencias publicadas sobre los resultados económicos de algunos procedimientos, como la resección prostática transuretral (3-5), que ha proporcionado una gran reducción de los costos y mayor eficiencia. Aunque los gastos por pago a profesionales (cirujano, anestesista) no cambian, los gastos de los servicios varían considerablemente, hasta el punto de que en una de las publicaciones mencionadas el costo de esta intervención fue seis veces menor en la modalidad ambulatoria que en la hospitalaria.

Un estudio reciente (6) ha comparado los resultados, los gastos y la satisfacción del usuario entre la cirugía convencional y la cirugía de día y la atención domiciliaria posterior en dos intervenciones de gran volumen -la herniorrafia y la colecistectomía laparoscópica-, comprobándose que esta nueva modalidad utiliza menos recursos y reduce significativamente la estadía. En ese estudio, con la cirugía de día y la atención domiciliaria posterior, los costos medios se redujeron en al menos \$US 385 para la herniorrafia y \$US 427 para la colecistectomía laparoscópica. Otros trabajos recientes sobre la herniorrafia laparoscópica (710) señalan ventajas, pero también recomiendan precaución y aconsejan la realización de nuevos estudios antes de proceder a la adopción amplia de esta modalidad, por faltar aún una evaluación clínica y de costos a gran escala.

Entre las especialidades incluidas en este estudio, las que mejores resultados proporcionaron fueron la cirugía general, la cirugía plástica y la oftalmología. Para las otras especialidades el resultado fue menor, posiblemente por tratarse de situaciones en las que han incidido factores variados, como los problemas organizativos dentro del departamento o área (por ejemplo, en cirugía pediátrica) o la au- 
sencia de definición clara de los criterios y del plan de inclusión de enfermedades o técnicas (por ejemplo, en ORL y urología).

Una de las limitaciones de este estudio radica en el hecho de que no se incluyera la cirugía ginecobstétrica (legrados, conizaciones, cirugía laparoscópica), por realizarse en otra unidad sanatorial. En caso de que se hubieran incluido estos procedimientos, seguramente se hubieran obtenido mayor cobertura de la nueva modalidad y mejores resultados en el análisis de costo-efectividad.

Por otra parte, esta modalidad de organización del trabajo quirúrgico produce una disminución de las listas de espera para la intervención y un aumento de la posibilidad de realizar intervenciones quirúrgicas de forma oportuna, sin complicaciones, sin mortalidad y con un bajo porcentaje de reingresos (11). La disminución de las listas de espera fue notable en esta institución en el caso de la cirugía general y la urología.

En cuanto a la evaluación del costoefectividad, la forma de presentación del presupuesto de la actividad en la institución fue una limitante que hubo que sortear, dado que no existía un presupuesto unificado de todos los gastos de la nueva modalidad. Se recurrió a todas las fuentes de datos existentes a fin de calcular el costo-día de atención y se identificó como necesidad la reformulación del sistema de información de intervenciones quirúrgicas y sus costos para adaptarlos a las nuevas formas de organización. Los recursos que se incorporaron como inversión inicial fueron bajos. Los gastos de esta nueva forma de funcionamiento son menores que en la cirugía convencional, lo cual convierte a aquella en una de las nuevas modalidades de organización asistencial con mejor relación costo-efectividad.

Los resultados de este estudio fueron similares a los obtenidos en países como España (12), con procesos similares a los incluidos en nuestro medio. Hay avances aun más marcados en países como Alemania (13), dentro de un sistema de atención diferente.

La aplicación de esta nueva modalidad de atención quirúrgica en intervenciones seleccionadas de cirugía general y especialidades permitió reducir en 1,5 días la internación hospitalaria por intervención, lo cual significa que tiene una relación costoefectividad dos veces y media mejor que la modalidad tradicional. Así pues, esta evaluación de la UCD del CASMU permite recomendar definitivamente la adopción y desarrollo de esta forma de trabajo en las áreas ya abordadas y su ampliación hacia otras áreas de la cirugía. Esta forma de atención reduce radicalmente los costos, sobre todo debido a la disminución de la estadía hospitalaria y de los recursos destinados a los servicios de internación en cama hospitalaria, con un alto grado de satisfacción del paciente e indicadores de buena calidad de la asistencia, en lo cual coincide con la experiencia internacional (14-16).

\section{REFERENCIAS}

1. Drummond M, Mason J. Biotechnology in the changing health-care environment: methods for economic evaluation of innovative technology. En: The economic aspects of biotechnologies related to human health. Part 1: Biotechnology and medical innovation: socioeconomic assessment of the technology, the potential and the products. Paris: OECD; 1997. Disponible en: http://www.oecd.org/ pdf/M000014000/M00014590.pdf. Acceso el 26 agosto 2002.

2. Brennan PF. Patient satisfaction and normative decision theory. J Am Med Inform Assoc 1995;2:250-259.
3. Klimberg IW, Locke DR, Dersch MW, Taub HC. TURP as same-day surgery. Contemporary Urology; nov 1996. Disponible en: http://www.contemporaryurology.com/ be_core/ $\mathrm{u} /$ sections/c_past_issues / index. jsp? navty $\mathrm{pe}=\mathrm{u} \&$ show $\mathrm{Poll}=$ no\&filename $=$ $<$ filename/>. Acceso el 26 agosto 2002.

4. Gheiler EL, Lovisolo JA, Tiguert R, Teifilli MV, Grayson T, Olford G, et al. Results of a clinical care pathway for radical prostatectomy patients in an open hospital-multiphysician system. Eur Urol 1999;35:210-216

5. Palmer JS, Worwag EM, Conrad WG, Blitz BE, Chodak GW. Same day surgery for radical
En el área de la cirugía ambulatoria, el desafío actual parece consistir en consolidar condiciones favorables para lograr niveles óptimos de calidad en la atención y procurar ampliar las intervenciones incluidas.

Agradecimientos. Se agradece la participación, colaboración y apoyo de las autoridades e integrantes de la Dirección del CASMU y de todas las Jefaturas y Equipos Técnicos. Mencionamos especialmente a las siguientes personas que tuvieron un papel destacado en las actividades de planificación, ejecución y evaluación: los doctores Óscar Balboa, Juan Jacobo Hornblas, Salo Kurek, Martín Harreche, José Trotchansky, Juana Wettstein, Raúl Cepellini, Ángel Vivas, Roberto Masliah, Alberto Irigaray, Carlos Rosasco, Ana Sollazo, Milton Carro, Olga Caracha y Ana Pérez, las licenciadas en enfermería Nelly Fernández, Gladys Herrera y Cristina Guerra, los arquitectos Aldo Acereza, Omar Olaizola y Susana Cora, el ingeniero Jorge Di Fabio, el psicólogo Carlos Cavasín y el personal técnico, administrativo y de servicio asignado a la Unidad de Cirugía de Día y al Departamento de Registros Médicos. Además, agradecemos especialmente el asesoramiento en la realización de este trabajo por parte de Nelly Murillo y Dante Giménez, y la colaboración del Departamento Contable del CASMU (contadoras Luisa Otero y Carmen Alonso, y Ana Carpentieri). retropubic prostatectomy: is it an attainable goal? Urology 1996;47:23-28.

6. Board N, Caplan G. Implications of decreasing surgical lengths of stay. Aust Health Rev 2000;23:62-76.

7. McCormack K, Scott NW, Go PMNYH, Ross S, Grant AM, on behalf of the EU Hernia Trialists Collaboration. Laparoscopic techniques versus open techniques for inguinal hernia repair. The Cochrane Library, Issue 3. Oxford: Update Software Ltd.; 2002.

8. Lawrence K, McWhinnie D, Goodwin A, Doll $\mathrm{H}$, Gordon J, Gray A, et al. Randomised controlled trial of laparoscopic versus open repair 
of inguinal hernia: early results. Br Med J 1995;311(7011):981-985.

9. Lawrence K, Mc Whinnie, Goodwin A, Gray A, Gordon J, Storie J, et al. An economic evaluation of laparoscopic versus open inguinal hernia repair. J Public Health Med 1996;18:4148.

10. Farinas LP, Griffen FD. Cost containment and totally extraperitoneal laparoscopic herniorrhaphy. Surg Endosc 2000;14:37-40.

11. Bartlett MK, Battit GE, Rockett AM, Perry EJ, Austen MG. The role of surgery on ambulatory patients in one teaching hospital. Arch Surg 1979;114:319-320.
12. Botella JA, Martínez A, Uribe N, Valero J, Pardo J, Ruiz J. Ambulatory surgery: is a multidiscipinary unit necessary? Br J Surg 1997; 84:124-125.

13. Busse R, Schwartz FW. Financing reforms in the German hospital sector: from full cost cover principle to prospective case fees. Med Care 1997;35(10 Suppl):OS40-49.

14. Towle A. Changes in health care and continuing medical education for the 21st century. Br MedJ 1998;316:301-304.

15. Weisbrod BA. The health care quadrilemma: an essay on technological change, insurance, quality of care an cost containment. J Econ Lit 1991;29:523-552.

16. Davis JE. Major ambulatory surgery of the general surgical patient. Management of breast disease and hernia of the abdominal wall. Surg Clin North Am 1987;67:733-760.

Manuscrito recibido el 8 de febrero de 2002. Aceptado, tras revisión, el 15 de julio de 2002.

ABSTRACT Objective. To evaluate the Day Surgery Unit (DSU) of the Medical Union of Uruguay Health Center (MUUHC), in Montevideo. The DSU is located in the MUUHC's Sanatorium 1 hospital, a 107-bed hospital that had previously handled low- and medium-

Evaluation of a day surgery unit complexity surgeries. The results of the reorganization of the surgical activity were analyzed in terms of service utilization, costs, and patient satisfaction.

Methods. For four surgical specialties that were selected for study, health care data were gathered and used to determine the coverage level with the new approach, the number of days of hospitalization, the costs before and after putting the DSU into place, and the patients' opinions of the quality of the care.

Results. For the 1998-1999 period, which was the second full year of operation of the DSU, the DSU was carrying out more than a third of the surgeries of the four surgical specialties being studied. These four specialties accounted for $84 \%$ of the total number of surgeries carried out by the DSU in the 1998-1999 period. The total number of days of hospitalization declined by $26 \%$. The cost-effectiveness analysis showed that the conventional approach, with hospitalization, would have cost some two and a half times as much as the day surgery approach for the same surgeries. In general, the patients expressed strong satisfaction with the services they received from the DSU.

Discussion. Our results indicate that it is definitively possible to recommend this day surgery form of care, since it provides good quality of care, it satisfies patients, and it dramatically lessens costs, thanks to the reduction in hospitalizations and the resources needed for inpatient services. It may be possible to expand this new regimen by including other conditions and surgical techniques that are appropriate for day surgeries.

\section{Cirugía en el hospital de distrito: obstetricia, ginecología, ortopedia y traumatología}

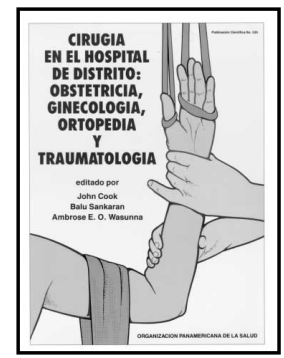

$1992 \cdot 240 \mathrm{pp}$.

ISBN 9275315353

Código: PC 535

Precio: \$US 28.00/ \$US 21.00 en América Latina y el Caribe
Este manual es una guía de gran utilidad para los médicos que prestan servicios quirúrgicos y de anestesia en hospitales pequeños con limitaciones de personal, equipo y fármacos, y en donde los médicos tienen poco acceso a los servicios de especialistas. El manual tiene numerosas ilustraciones detalladas y se divide en dos partes: 1) Obstetricia y ginecología. Aqui se describen los procedimientos obstétricos considerados esenciales para las complicaciones más importantes del embarazo y el parto, los procedimientos obstétricos básicos para prevenir la mortalidad materna y los procedimientos ginecológicos apropiados para el hospital de distrito. 2) Ortopedia y traumatología. Esta parte cubre las técnicas ortopédicas básicas, como la tracción, la aplicación de yesos y el tratamiento de fracturas específicas, luxaciones y otras lesiones, incluidas las quemaduras. 